\title{
Optimization for Onshore Wind Farm Cable: Connection Layout using ACO-AIA algorithm
}

\author{
Mohamed Tifroute* and Hassane Bouzahir \\ Laboratory of Engineering Systems and Information Technologies \\ ENSA, UIZ, PO Box 1136, Agadir, Morocco \\ *Corresponding author: mohamed.tifroute@gmail.com
}

Article history

Received: 16 November 2017

Received in revised form: 14 May 2018

Accepted: 17 May 2018

Published on line: 1 April 2019

\begin{abstract}
The wind farm layout optimization problem is similar to the Steiner Minimal Tree Problem (SMTP) of a weighted undirected graph. Due to the cable current-carrying capacity limitation, the cable sectional area should be carefully selected to meet the system operational requirement and this constraint should be considered during the SMTP formulation process. Hence, the traditional SMTP algorithm cannot ensure a minimal cable investment layout. In this paper, a hybrid algorithm based on modified Ants Colony Optimization (ACO) and Artificial Immune Algorithm (AIA) for solving SMTP is introduced. Since the Steiner Tree Problem is NP-hard, we design an algorithm to construct high quality Steiner trees in a short time which is suitable for real time multicast routing in networks. After the breadth - first traversal of the minimal graph obtained by ACO, the terminal points are divided into different convex hull sets, and the full Steiner tree is structured from the convex hull sets partition. The Steiner points can be vaccinated by AIA to get an optimal graph. The average optimization effect of AIA is shorter than the minimal graph obtained using ACO, and the performance of the algorithm is shown. We give an example of application in optimization for onshore wind farm cable. The possibility of using different sectional area's cables is considered in this paper.
\end{abstract}

Keywords Steiner problem; ant colony algorithm; artificial immune algorithm.

Mathematics Subject Classification 05C05, 52A21, 05C76, 9OC15.

\section{Introduction}

In general, the location of wind farms is usually far away from energy consumers. Consequently, power transmission is an important issue when planning wind farms.

A typical wind farm contains a group of wind turbines, substations and grid points which are connected by a cabling system (see Figure 1). The turbines are driven by wind wheels and convert wind's kinetic energy into electrical power. This electrical energy is transferred by the cabling system to a substation collecting the electrical power of a group of turbines. The substation transforms the electrical energy to alternating current with a high voltage level to 

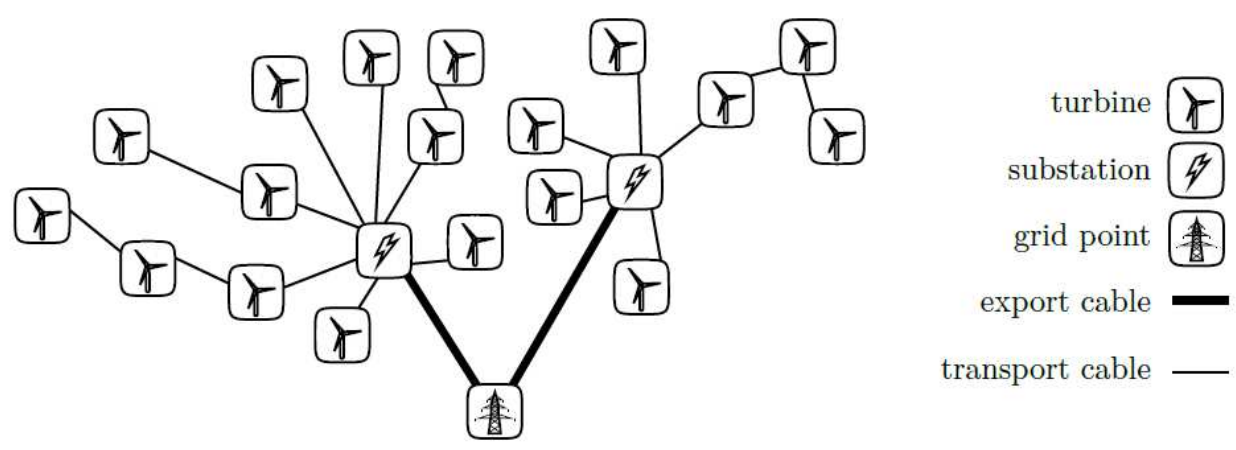

Figure 1: Structure of a Wind Farm

minimize transport energy loss. All substations are connected to a grid point, which is the entry of the general power grid.

When planning a wind park there is a variety of properties to consider. Most important in view of cost and energy production is positioning of the turbines and substations as well as the electrical infrastructure of the park. Finding an optimal wind farm layout is a very complex task that is like many other optimization problems such as the traveling salesman problem not solvable in polynomial time. Even the substation problem which contains finding a cabling between multiple turbines and a single substation is NP-hard as it is equal to the capacitated minimum spanning tree problem [1]. As a result wind farm projects today are often designed manually.

We limit the wind farm layout problem by considering the turbines and substations as objects with fixed positions. The remaining subproblem consists of finding an optimal cable system. We call this problem the wind farm cabling problem. As reported by Kirby et al. [2] such electric infrastructure accounts for approximately $15 \%$ of the total cost of an onshore wind farm. Different cabling layouts differ in costs and reliability through redundancy.

Ant colony optimization is a meta-heuristic optimization method that simulates the behavior of ants in a graph model. In every calculation step, an ant will be simulated to finds a random solution by choosing a path in the graph. Depending on the quality of this solution, a smaller or larger amount of pheromones will be dropped along the chosen path. The pheromone affects the random selection of the future ant. An ant will more likely choose a direction where more pheromones are dropped.

Ant colony optimization (ACO) is an algorithm that has been successfully applied to other optimization problems such as maximizing energy yield in the wind farm layout problem [3] which are both similar to our problem. This paper discusses the problem of designing a wind energy collection network that is part of a large class of network design problems. Apart from the complexity inherited from this class, the latter subject to an important technical constraint namely the non-bifurcation of energy. In fact, network designers require that the energy produced by a group of wind turbines and combined on a given link be combined until it reaches the substation. This problem is similar to the mathematical problem of finding the Minimal Steiner Tree (SMT) in an undirected weighted graph. Due to the limitation of the current carrying capacity of the cable, the cable section must be carefully selected to meet the operational requirements of the system and this constraint must be considered during the 
SMTP formulation process. Therefore, the traditional algorithm to find Steiner's minimal shaft can not minimize wiring costs.

Thus, we introduced a hybrid algorithm based on Modified Ant Colony Optimization (ACO) and Artificial Immunity (AIA) algorithm to solve SMTP. As the SMTP problem is NP-hard [4], our algorithm builds in a short time Steiner trees that are of high quality and suitable for real-time multicast routing in networks.

Our hybrid algorithm traverses the minimal tree obtained by ACO, the end points are divided into different convex subsets, Steiner's points are vaccinated by AIA in each of these sets, in order to obtain a more optimal graph. This hybridization allowed us to obtain a shorter routing than that obtained using the ACO algorithm.

\section{Problem Formulation}

\subsection{Cost Model}

The cost models are set up according to cables rated power. The mathematical equations can be written as follows [5]:

$$
\begin{aligned}
& C_{i}=A_{p}+B_{p} \exp \left(\frac{C_{p} S_{\text {rated }, i}}{10^{8}}\right)^{2} \\
& S_{\text {rated }, i}=\sqrt{3} I_{i, \text { rated }} U_{i, \text { rated }}
\end{aligned}
$$

where $C_{i}[\mathrm{MMD} / \mathrm{km}]$ is unit cost of cable $i, A_{p}, B_{p}$ and $C_{p}$ are coefficients of cable cost

model. $S_{\text {rated, } i}, I_{i, \text { rated }}$ and $U_{i \text {,rated }}$ are respectably, rated apparent power, rated current and rated voltage of a cable in line $i$.

After the voltage level is decided, the cables are selected according to their rated current which is correlated to the sectional area. The cable types are selected according to an existing cable list in [5]. In some cases, more cables are required between two Wind Turbines (WTs) if many are connected after a branch.

In Table 1, the parameters that are used in this work for the Alternative Current (AC) cable cost model are shown for the different rated voltages. As can be noticed from Equation (1), the cost of the AC cables increases exponentially with the rated power of the cable [5].

Table 1: Cost Parameters for AC Cables for Different Voltages

\begin{tabular}{|c|c|c|c|}
\hline Rated voltage $[\mathrm{kV}]$ & $A_{p}\left[10^{6}\right]$ & $B_{p}\left[10^{6}\right]$ & $C_{p}$ \\
\hline 22 & 0.284 & 0.583 & 6.15 \\
\hline 33 & 0.411 & 0.596 & 4.1 \\
\hline 45 & 0.516 & 0.612 & 3 \\
\hline 66 & 0.688 & 0.625 & 2.05 \\
\hline 132 & 1.971 & 0.209 & 1.66 \\
\hline 220 & 3.181 & 0.11 & 1.16 \\
\hline
\end{tabular}




\subsection{Objective Function}

If the location of WTs and Substation regarded as vertices while the costs of the cables are regarded as the weight of branches, then, the problem then can be converted into a classical mathematical problem as finding the Minimal Steiner Tree (MST) of a given graph. Therefore, the mathematical expression of the problem can be written as follows [5]:

Objective function:

$$
\text { Cost }_{\min }=\min \left(C_{i}^{\left(G_{T}\right)}(x, y) L_{i}^{\left(G_{T}\right)}(x, y)\right) ; G_{T} \in G
$$

Constraints:

$$
\begin{gathered}
I_{i} \leq I_{i, \text { rated }} ; i \in\{1, \ldots, N-1\} \\
x \in\left(0, L_{x}\right) ; y \in\left(0, L_{y}\right)
\end{gathered}
$$

As mentioned before, $G$ is an undirected weighted graph, which represents all possible cable connection layouts. $G_{T}$ is one layout of $G$ which contains a number of vertices ( $V$ : WT location) with different weights ( $W$, cable cost) between them. $N$ which is the total number of WTs plus Substation in this work. Cost min $[\mathrm{MMD}]$ is minimum cost of total cables for optimized cable connection layout, $L_{x}, L_{y}$ are predefined area for constructing Substation, respectively, in $x$ direction and $y$ direction and $I_{i}$ is current flowing through cable $i$. Once the tree shape layout $G_{T}$ is determined, the total cost of cables can be calculated using (1)-(5).

\section{Minimal Steiner Tree Problem}

Let $D:=\left\{a_{i}\right\}_{i=1, \ldots, n}$ be a given set of points in the Euclidean plane. A natural task, which arises in many optimization problems, is finding a connected graph with minimal length whose set of vertices is $D$. If we are not allowed to add other vertices to the graph, the solution is called a minimum spanning tree (MST). Finding such a solution is a purely combinatorial problem. One can tackle the problem by considering the complete tree (i.e., the tree containing all possible edges with a set of vertices $D$ and weights on the arcs given by the distance between the corresponding vertices. Then, one is asked to find a connected subgraph of the given weighted graph, which contains all the vertices and which has a minimal length. The problem becomes much more difficult if one has the possibility to add new vertices to the given set of points. This variant of the problem is called the Steiner problem [6]. It means finding a connected graph with minimal length among all graphs whose set of vertices contains the given set of points. The additional vertices are called the Steiner points.

The mathematical formulation of the problem can be written as follows

$$
\min \left\{\mathcal{H}^{1}(K) ; K \subset \mathbb{R}^{2} \text { compact, connected, and containing } D\right\}
$$

with $\mathcal{H}^{1}(K)$ the one-dimensional Hausdorff measure of $K$.

The objective of the Euclidean Steiner Tree Problem (ESTP) is to determine the minimal length tree (with respect to the Euclidean metric) spanning a set of terminal points, while permitting the introduction of extra Steiner points $S$ into the tree to reduce its overall length (see [6]). A topology is a configuration of terminal points and Steiner points where the connections are specified, but the locations and number of the Steiner points are not. A topology is said to be a Steiner topology if every Steiner point has degree 3 and every terminal node has degree maximum 3. A Steiner topology where all terminal points have degree 3 is 
a Full Steiner Topology (FST). Any non-FST can be identified with a FST where some edges have zero length. Such an FST is called degenerated. The cause of degeneration is due to the distribution of terminal points. A non-FST can be decomposed into some FST's. A solution to the ESTP is called a Steiner Minimal Trees (SMT). It can be shown that an SMT has a Steiner topology. Other known results regarding the SMT include the following (see [8])

- The angle condition: the angles between edges connecting a Steiner point and its 3 neighbors are all 120 degrees.

- Any SMT is a concatenation of FSTs on subsets of terminal points.

- Any SMT for a problem on N terminal points has at most N-2 Steiner points.

\section{Minimal Network Route Construction Using the Ants Colony Optimization Algorithm}

Ants live together in colonies and they use chemical cues called pheromones to provide a sophisticated communication system. An isolated ant moves essentially at random but an ant encountering a previously laid pheromone will detect it and decide to follow it with high probability and thereby reinforce it with a further quantity of pheromone. The repetition of the above mechanism represents the collective behaviour of a real ant colony which is a form of autocatalytic behaviour where the more the ants follow a trail, the more attractive that trail becomes. The above behaviour of real ants has inspired ACO which has proved to be an effective metaheuristic technique for solving many complex optimization problems [9]. This technique uses a colony of artificial ants that behave as cooperative agents in a mathematical space where they are allowed to search and reinforce pathways (solutions) in order to find the optimal ones. The feature of artificial ants is: a memory, not being completely blind and the process time is discrete.

The solution uses a graph with $n$ vertex. Each edge $(i, k)$ of a complete graph is associated with a pheromone, concentration $\left(\tau_{i k}\right)$ used for the chosen route of the ant in the colony. Initially, $\tau_{i k}$ is set to small positive value (i. e., 0.01). In the ACO algorithm, the minimum route length was initialized with a high value symbolically denoted $L_{\text {min }}=\infty$. The number of ants $n a$ will be distributed as evenly as possible between the graph vertices. The number of vertices and the number of ants are chosen such that $n a=m$ ( $m$ is integer), while the ants are will distribute uniformly in each node. Also, the place where each ant $(j)$ is located at one time is represented by $\operatorname{Nod}_{j}$. According to the proposed problem the optimization process contains the restriction that an ant must pass through each node without forming cycles. Each ant's route selection is done in tabu list, which contains the elements that describe the sequence of visited nodes (vertices). After the distribution of ants in the graph nodes, the tabu list assigned to each ant will initialize the first position with the order number of the node where that ant was distributed.

Further, the ants should move in different graph nodes, until the tabu lists are complete,

each ant making a complete graph tour. For every ant $j$, the starting node $i=\operatorname{Nod}_{j}$ and destination node $k^{*}$ are considered. The $k^{*}$ should not be included in the set of sessions that remain to be observed by ant $j$ positioned at session $i$. The probability for an ant $j$ in a node 
$i$ to choose node $k$ as its destination is defined as follows:

$$
P_{i k}=\left\{\begin{array}{ll}
\frac{\left(\tau_{i k}\right)^{\alpha} \cdot\left(\eta_{i k}\right)^{\beta}}{\sum_{p \notin T a b u_{j}}\left(\tau_{i p}\right)^{\alpha} \cdot\left(\eta_{i p}\right)^{\beta}}, & k \notin T a b u_{j} \\
0, & k \in T a b u_{j}
\end{array} .\right.
$$

$\operatorname{Tabu}_{j}(i)$ is the set of sessions that remain to be observed by ant $j$ positioned at session $i$. It is shown that the quality of the path $(i, j)$ is proportional to its shortness and to the highest amount of pheromone deposited on it (i.e., the selection probability is proportional to path quality), $\tau_{i k}$ represents the amount of pheromone in the edge $i k$ and visibility $\eta_{i k}$ probability. If $\beta=0$, the $P_{i k}$ probability depends on pheromone concentration. Also, if $\alpha=0$ this probability depends only on the nodes visibility (distance between nodes). When all the ants have passed through all the graph nodes, each ant route is closed without returning to the origin node. Practically, this aspect is the ACO algorithm adaptation to the studied problem. Further, according to the ACO, the route lengths for all ants' must be calculated and the minimum length is stored, which coincides with the final iteration. Before switching to another step, the pheromone concentration must be updated on each graph edge by using:

$$
\tau_{i k} \leftarrow(1-\rho) \tau_{i k}+\sum_{j=1}^{m} \Delta \tau_{i k}^{j} .
$$

Later, in the evaporate pheromone phase, pheromone trails are evaporated in the following formula:

$$
\tau_{i k}=\rho \cdot \tau_{i k}+\Delta \tau_{i k}
$$

Where $\rho$ is a parameter set to 0.1 in most of the experiments, $\Delta \tau_{i k}$ represents the pheromone concentration correction on the edge $(i, k)$ determined by total ant number who move from $i$ to $k$, using the equation:

$$
\Delta \tau_{i k}=\sum_{j=1}^{m} \Delta \tau_{i k}^{j}
$$

where $\Delta \tau_{i k}^{j}$ represents the deposited pheromone quantity on edge $(i, k)$ by ant $j$, determined as follows:

$$
\Delta \tau_{i k}^{j}(t)= \begin{cases}\frac{Q}{L_{j}(t)}, & k \in \operatorname{Tabu}_{j}, i=\operatorname{Tabu}_{j}(p) ; k=\operatorname{Tabu}_{j}(p+1) \\ 0, & \text { otherwise, }\end{cases}
$$

with stopping criterion coincides with maximum number of iterations $\left(T_{\max }\right)$. While $t<T_{\max }$, the reset tabu lists of the ants and the procedure is restarted by resetting the first element of every tabu list with current node number where each ant is located. ACO algorithm is versatile and robust.

\section{Artificial Immune Algorithm}

The AIA is a recent branch of stochastic search algorithms [7]. It is classified as a populationbased metaheuristic method. Many authors claim that AIA is a more efficient problem-solving 
strategy in optimization. The original intention is inspired by the simulation of the physiological immune systems of natural living organisms that defends the body from foreign pathogens (bacteria or virus). The mechanisms work by first recognizing foreign substances known as antigens. Immune systems then generate a set of antibodies to eliminate the antigens. The mechanisms are able to recognize which antibodies are better at eliminating the antigens and thus produce more variations in the next generation of antibodies. Each antibody is assigned a value called affinity the latter shows the capability of that antibody to eliminate antigens. The antigen and affinity in the AIA are respectively equivalent to the problem to be solved or the objective function and the feasible solution for a conventional optimization method. The terminlogy of AIA are:

- Antigen: The problem to be solved, i.e., the objective function.

- Antibody: Solutions for problem to be solved.

- Vaccine: Feature information extraction from the prior knowledge of the problem to be solved.

- Fitness function: Judgment of individuals in the population quality index.

\section{Immune Algorithm for Solving SMT}

A non-FST can be decomposed into some full Steiner trees. Which terminal point appears in full Steiner tree, it is the most critical issue. The Steiner points are inoculated as vaccine to a minimal tree obtained using ACO. The steps that give the final solution are as follows.

\section{Procedure: Inoculation of Steiner points using AIA.}

Step 1 Dividing the Minimal Tree Obtained using ACO into Different Convex Hull Sets For the terminal points set $D=\left\{a_{1}, a_{2}, \ldots, a_{n}\right\}$ in the plane, first, the minimal tree is generated using ACO. Then, according to the order of the breadth-first search, our algorithm crosses the minimal tree and the nodes are divided into different subsets. In the same convex hull sets with nodes belonging to a collection, the ones on the segmentation may appear into two different subsets (Figure 2).

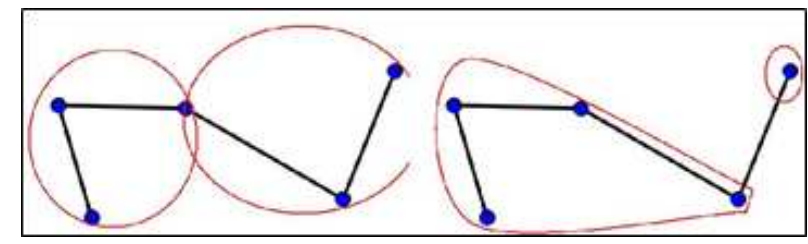

Figure 2: Two Different Partition Convex Hull Parts of 5 Points

In the division of a convex hull, one has to respect the following principles:

- To ensure that carving up the minimal tree obtained by ACO can get maximum number of Steiner points.

- To minimize numbers of terminal points not in full in the topology structure. 


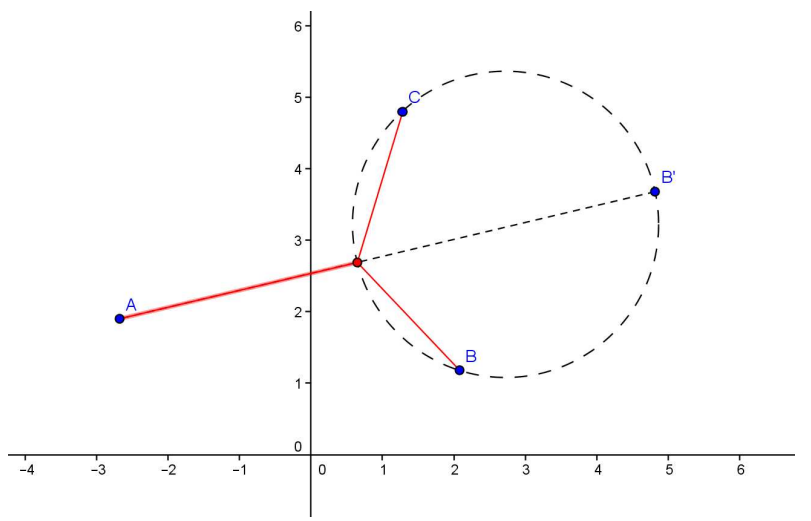

Figure 3: Vaccines of 3 Points in a Convex Hull

- To have the maximum number of convex hull sets.

Step 2 Analysis of the Problem, Collection of Feature Information, Gain of Vaccine
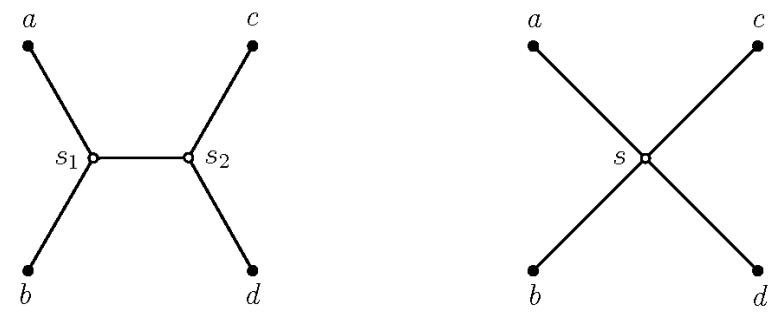

Figure 4: FST for 4 Points

For the convex set, find Steiner points using the method of Melzak [10], then extract the Steiner point as vaccine. Three points full Steiner topology is one. Four points full Steiner topology is two or one, as in Figure 4.

Step 3 Inoculation in the Minimal Tree Obtained using ACO

The extracted vaccines join the minimal tree obtained by ACO. Given sets $V\left(a_{i}\right)$ of
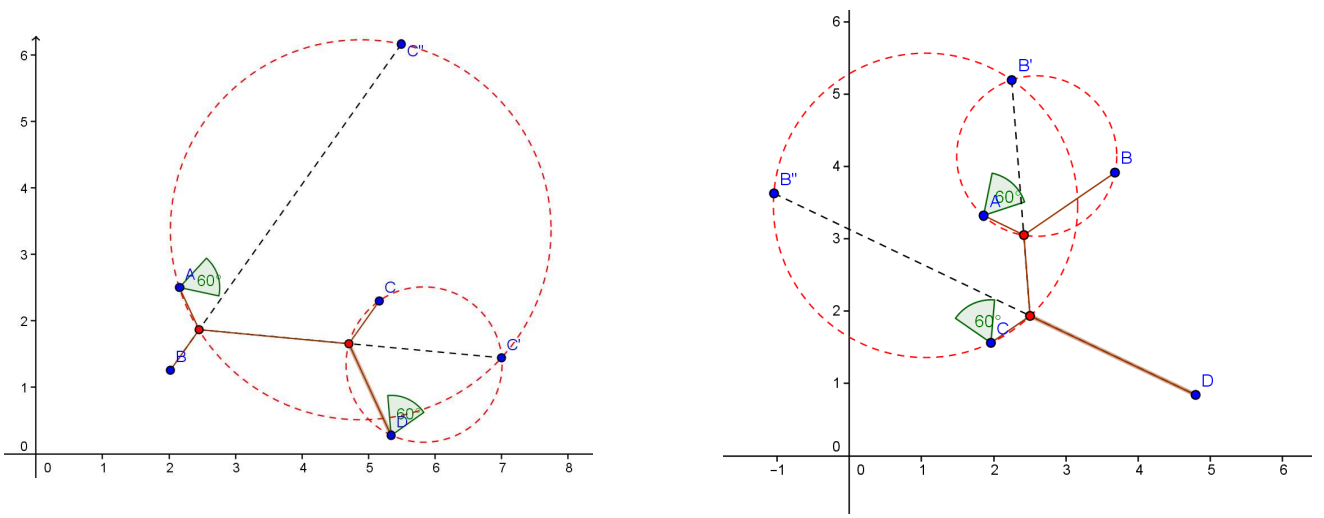

Figure 5: Two Different Vaccines of 4 Points

terminal points, $E\left(e_{i}\right)$ correspond to the edges set of the minimal tree obtained by ACO, $G\left(g_{i}\right)$ is the edges set of the obtained Steiner tree after vaccination. 
(a) $L(A C O)=\sum_{i=1}^{n-1} e_{i}$

(b) $L(S M T)=\sum_{i=1}^{m} g_{i}$;

(c) $f(v)=\frac{L(S M T)}{L(A C O)}$

\section{Algorithm}

Step 1 The initial set $V$ gets minimal tree obtained by ACO. Compute length and consider this minimal tree as the initial Steiner tree solution.

Step 2 Make all possible partitions as a convex hull.

Step 3 Extract all kinds of vaccines of a convex hull and get a set of vaccines.

Step 4 Select some vaccines to vaccinate in initial solution and calculated Steiner tree length, and delete this vaccines from the set of vaccines.

Step 5 Reckon fitness function. If the value of fitness function is shorter than before, save this inoculation, or else cancel.

Step 6 If $f(v)<0.87$ or the set of vaccines is null, algorithm end, or else go to Step 4 .

\section{Optimization for Onshore Wind Farm Cable}

\subsection{Optimized Connection Layout without Considering Rated Current of Cable}

Radial or branched wind farm cable design is divided into two phases: one uses ACO algorithm building the minimal cable route length and the second improves the network by adding supplementary branch nodes using AIA. The wind farm structure or cable route improvement can be achieved by shifting the source nodes to the ends and vice versa, with the particularity that the latter would be preferable because the ends power flows are known. By using the method above, farm arborescence cable route optimization application can be designed. The allocation uses a combination of ACO and AIA and the objective function is used to obtain a minimal cable route length (between wind turbines and the substation).

Our objective is to follow a hybrid approach that gives the optimal cable route for a wind farm with 26 wind turbines. The input data of the test are presented in Figure 6. As a first step, we use the aforementioned methodology that is based on the ACO successive search technique. The obtained minimal length for the wind farm cable route is $16700 \mathrm{~m}$. This configuration is shown in Figure 7. The second step is to allow a new route construction. 14 Steiner points are added by AIA (see Figure 8) and the minimal wind farm cable route length is reduced to $15040 \mathrm{~m}$ (see Figure 9) [11]. The obtained output data are shown in Table 2.

Table 2: Output Data [11]

\begin{tabular}{|c|c|c|c|c|c|}
\hline Turbines & Convex hull & Vaccines & L(ACO) & L(SMT) & Ratio \\
\hline 26 & 13 & 14 & $16700 \mathrm{~m}$ & $15040 \mathrm{~m}$ & 0.9 \\
\hline
\end{tabular}




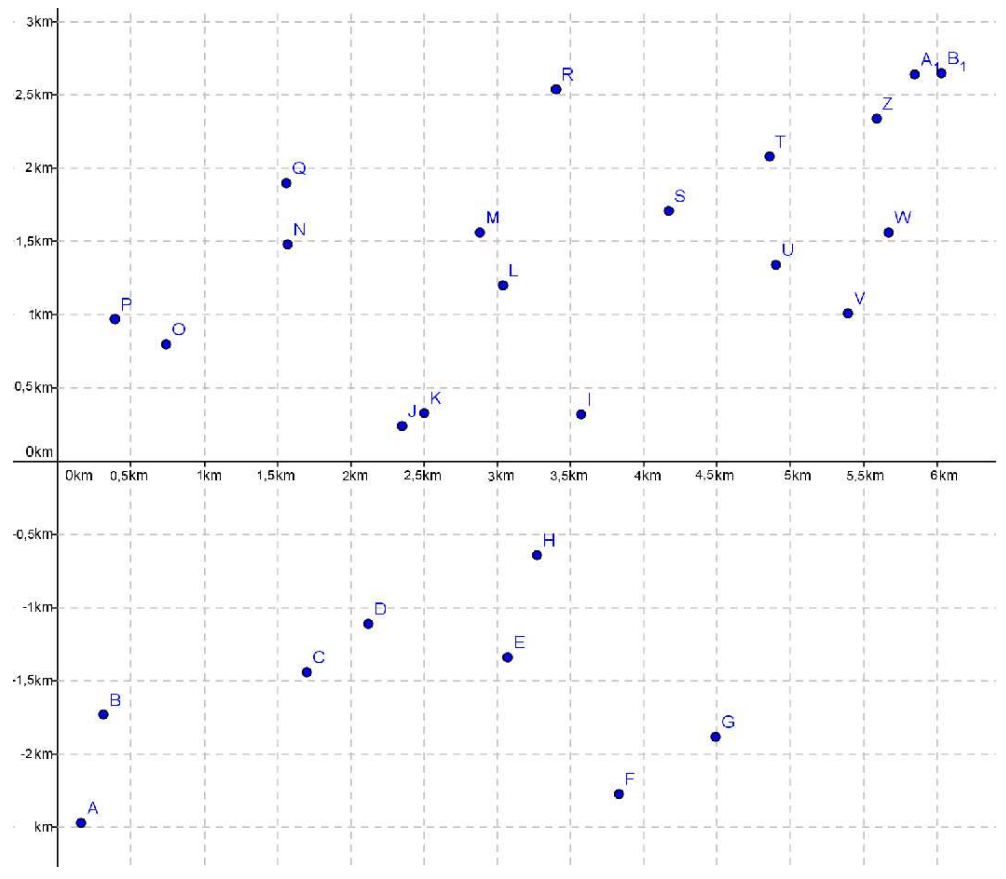

Figure 6: Localisation of Wind Turbines in the Analyzed Wind Farm

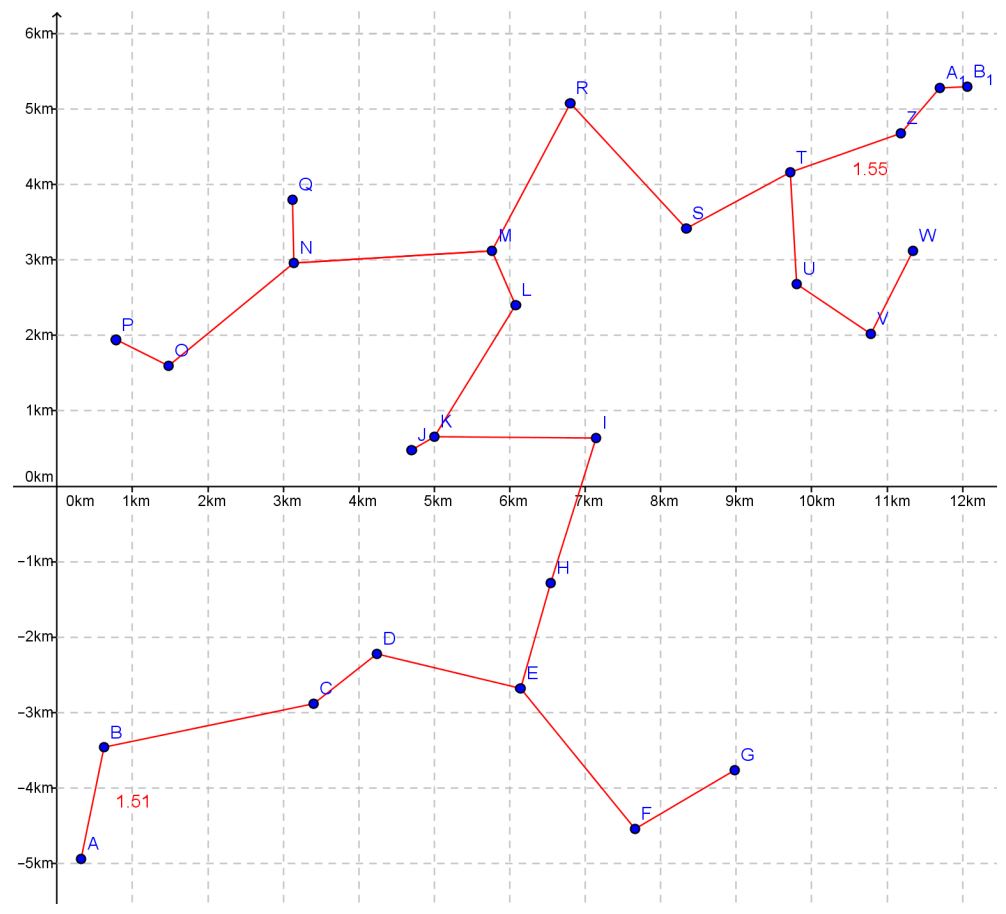

Figure 7: Wind Farm Cable Route Optimization Using ACO Algorithm 


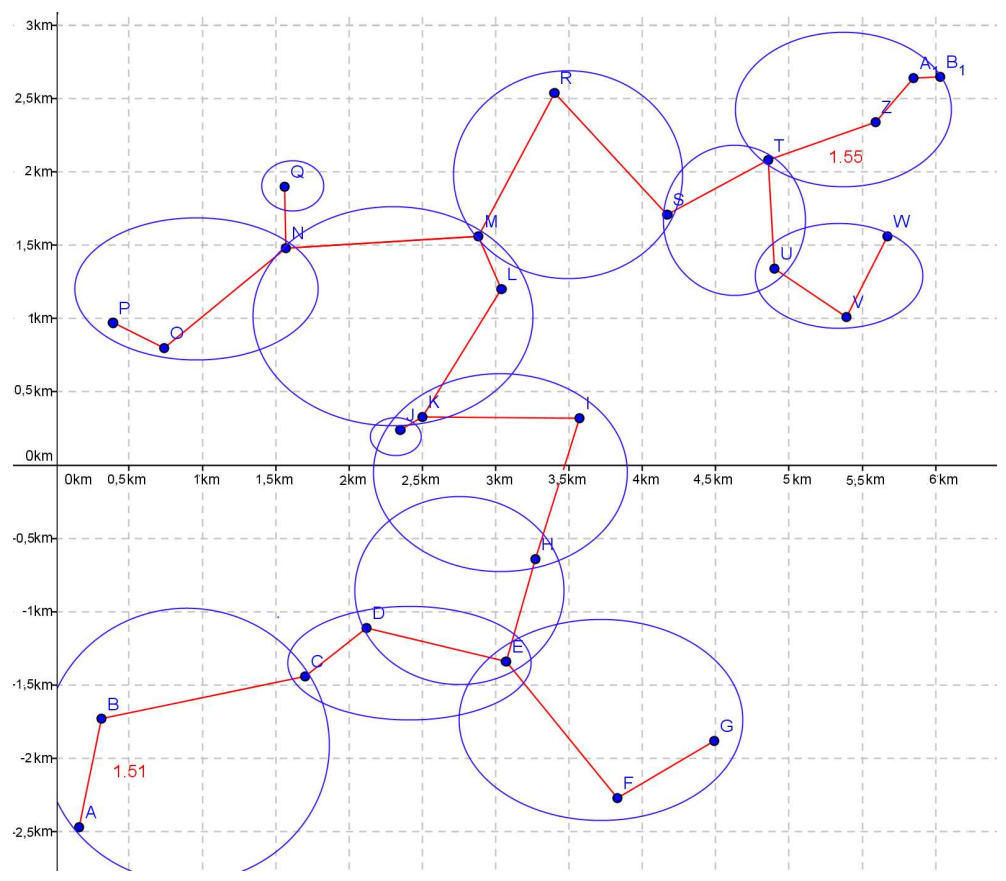

Figure 8: Optimal Convex Hull Partition

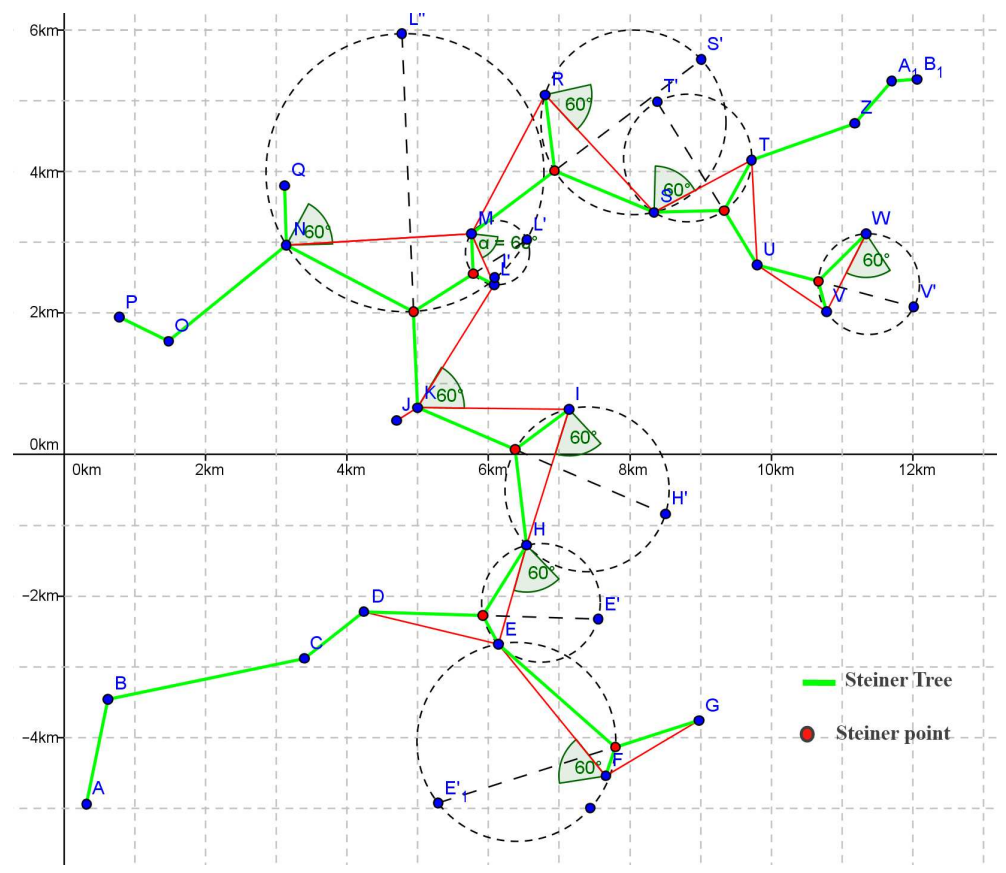

Figure 9: SMT Obtained Using AIA 


\subsection{Calculations for the Choice of Cables}

It is necessary to define the distribution of wind turbines by clusters (i.e., groups of wind turbines interconnected with each other and with the evacuation station). The diameter of each section is determined and verified for two key parameters:

- The permissible current for the section

- The voltage drop for the entire cluster.

The permissible current is based on the maximum current for the limit cases of $\cos (\varphi)=0.9$ with $\varphi$ is the phase angle. The voltage drops the case of the normal operation by $5 \%$. The calculation of the maximum current flowing through each of the segments is as follows

$$
I=\frac{\text { Number of turbines per segment } \times P}{\sqrt{3} \cos (\varphi) \times U} .
$$

The minimum cable size that complies with the current density is chosen from the cable table. Then, both the individual voltage drop is calculated using the following formula

$$
V_{\text {drop }}=\frac{\sqrt{3} \times \text { length } \times I \times(R \cos (\varphi)+X \sin (\varphi)}{V} \times 100 \%
$$

with the resistance $R$ of a conductor (conduction ability) is given by the formula $R=\frac{R . \rho}{S}$ where $\rho$ represents the resistivity, $L$ is the length of cables and $S$ is the section. $X$ is the linear reactance in $\Omega / \mathrm{km}$. The maximum permissible currents for soil temperatures of $20 \mathrm{C}$ are in Table 3.

Table 3: Maximum Permissible Currents for Soil Temperatures of $20^{\circ} \mathrm{C}$

\begin{tabular}{|c|c|}
\hline Iteration cable sectional area $\left(\mathrm{mm}^{2}\right)$ & $I_{\max .}$ at $20^{0} \mathrm{C}[\mathrm{A}]$ \\
\hline 120 & 300 \\
\hline 240 & 440 \\
\hline 300 & 500 \\
\hline 500 & 640 \\
\hline
\end{tabular}

For Morocco, ambient and ground temperatures for a period of several months are higher than the standard temperatures specified in the UTE guide $\left(20^{\circ} \mathrm{C}\right.$ land $)$. The correction factors according to UTE C must be applied in order to arrive at the permissible current in the cables taking into account the actual temperature, the thermal conductivity of the ground and the type of cable arrangement that can be laid in clover or horizontal plane etc.. The maximum operating temperature of the cable must be considered (Table 4). These chemical insulated cables (XLPE) can operate up to $90^{\circ} \mathrm{C}$.

Depending on the thermal resistivity of the soil, the correction factors in Table 5 are applied. Depending on the installation depth, the correction factors (Table 6) must be applied.

The calculation of the maximum current flowing through each of the segments must take into consideration the operating temperature of the cable (Table 4), the thermal resistivity of the soil (Table 5) and the installation depth (Table 6). 
Table 4: Correction Factors Depending on the Operating Temperature of the Cable

\begin{tabular}{|l|l|l|l|l|l|l|}
\hline Soil temperature in ${ }^{0} \mathrm{C}$ & 15 & 20 & 25 & 30 & 35 & 40 \\
\hline Correction factor & 1.04 & 1 & 0.96 & 0.93 & 0.89 & 0.85 \\
\hline
\end{tabular}

Table 5: Correction Factors Depending on the Thermal Resistivity of the Soil

\begin{tabular}{|l|l|l|l|l|l|l|}
\hline Thermal resistivity & 0.85 & 1 & 1.2 & 1.5 & 2 & 2.5 \\
\hline Correction factor & 1.07 & 1 & 0.93 & 0.85 & 0.75 & 0.68 \\
\hline $\begin{array}{l}\text { The class of soil according } \\
\text { to the classification of the } \\
\text { ONEE }\end{array}$ & $\begin{array}{l}\text { Normal } \\
\text { terrain }\end{array}$ & $\begin{array}{l}\text { Dry } \\
\text { land }\end{array}$ & & $\begin{array}{l}\text { Very } \\
\text { dry } \\
\text { terrain }\end{array}$ & & \\
\hline
\end{tabular}

Table 6: Correction Factors Depending on the Installation Depth

\begin{tabular}{|l|l|l|l|l|l|}
\hline Depth of installation in meters & 0.80 & 1.00 & 1.20 & 1.30 & 1.50 \\
\hline Correction factor & 1.04 & 1.03 & 1.01 & 1.00 & 0.98 \\
\hline
\end{tabular}

Table 7: Specifications of Cables Colors

\begin{tabular}{|l|l|l|l|l|l|}
\hline & \multicolumn{5}{|c|}{ Collection system } \\
\hline Voltage level & \multicolumn{5}{|c|}{$33 \mathrm{kV}$} \\
\hline Type & \multicolumn{4}{|c|}{ AC } \\
\hline Color & Blue & Green & Orange & Cyan & Black \\
\hline Cable sectional & 70,95, & 185,240, & 400,500 & 630,800 & 1000 \\
area $\left(\mathrm{mm}^{2}\right)$ & 120,150 & 300 & & & \\
\hline
\end{tabular}


The lines show the cable connection layout and the color of the line represents the rating of the cable as is explained in Table 7 . Since multiple cables might be adopted between some pairs of WTs, the number of cables utilized between each two WTs is indicated by different types of lines: a solid line (one cable), dash line (two cables), dash dotted line (three cables). This is shown in the upper right box of Figure 11. The colors and lines in the following figures have the same meaning in this work.

\subsection{Optimized Connection Layout Considering Rated Current of Cable}

In order to show the performance of the proposed method, the layout obtained by ACO algorithm and ACO-AIA algorithm are compared through two scenarios which are described below.

\section{Scenario I:}

The Substation is Close to the First Turbines As can be seen in Figure 10, the wind turbines and Substation location are represented in a coordinate system. The substation is assumed to be located close to the first turbines. The blue points are WTs and red square is a substation. The lines show the cable connection layout and the color of the line represents the rating of the cable as it is explained in Table 7 and Table 3.

\section{Scenario II:}

Central Substation Placement Layout In this scenario, the substation is assumed to be constructed in the middle of the wind farm. The optimized layout obtained using ACOAIA algorithm is shown in Figure 11.

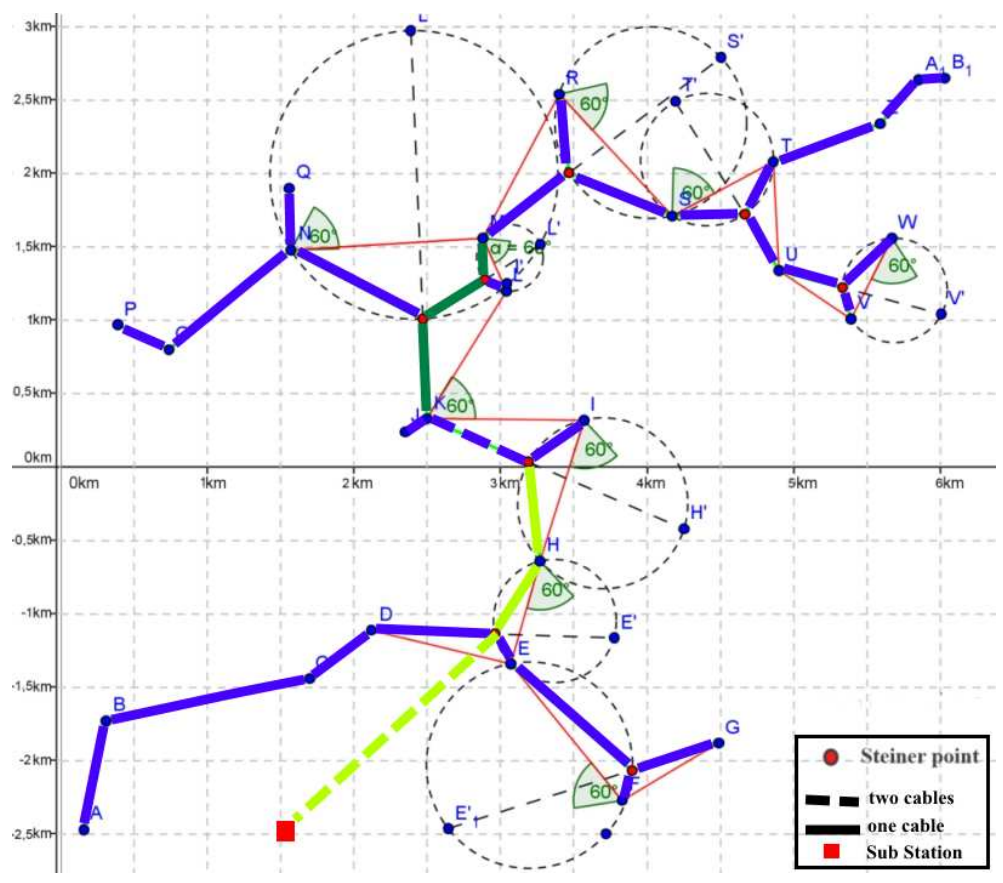

Figure 10: Scenario 1 


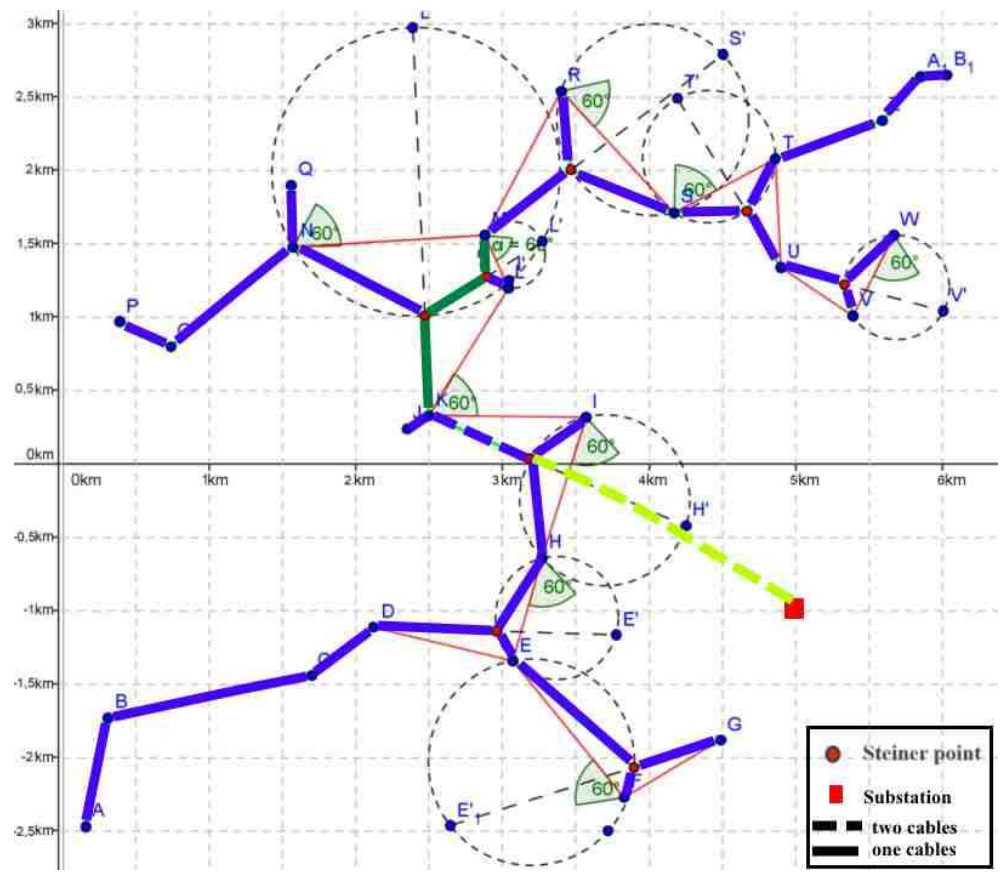

Figure 11: Scenario 2

Table 8: Comparison Results

\begin{tabular}{|l|l|l|}
\hline & Scenario I & Scenario II \\
\hline & ACO-AIA & ACO-AIA \\
\hline Total cable length for blue cable $(\mathrm{km})$ & 13.14 & 14.44 \\
\hline Total cable length for green cable $(\mathrm{km})$ & 1.243 & 1.243 \\
\hline Total cable length for orange cable $(\mathrm{km})$ & 1.08 & 0 \\
\hline Cable to substation $(\mathrm{km})$ & 1.702 & 1.774 \\
\hline Cable costs for blue cable (MMD) & 21.15 & 23.24 \\
\hline Cable costs for green cable (MMD) & 3.09 & 3.09 \\
\hline Cable costs for orange cable (MMD) & 3.451 & 0 \\
\hline Cable costs for cable to Substation (MMD) & 10.476 & 10.92 \\
\hline Total cable invest (MMD) & 38.57 & 37.25 \\
\hline Substation location (km) & $(1.5,-2.5)$ & $(5,-1)$ \\
\hline
\end{tabular}


It is shown in Table 8 that the ACO-AIA algorithm can find a cheaper layout. Comparing scenario I and scenario II, it can be seen that the total cost of cables is less in scenario II. The location of the substation has a significant impact on the total cable invest.

\section{Conclusion}

This paper proposes a new approach for wind farm electrical system design. The introduced approach is capable of finding the optimal wind farm cable route in a short time and with less computational effort comparing to exact methods, such as Kruskal and Prim Algorithms [12]. The possibility of using different cables sectional area is also considered in this paper. Our results show that the proposed algorithms can be used to generate a design that has a lower cost in terms of investment and energy.

\section{References}

[1] Aggarwal, Y. P., Aneja, V. and Nair, K. P. K. Minimum spanning tree subject to a side constraint. Computers and Operations Research. 1982. 9: 287-296.

[2] Kirby, L. L. M., Xu,N. M. and Siepmann, W. Hvdc transmission for large offshore wind farms.Power Engineering Journal. 2002. 16: 135-141.

[3] Eroglu, Y. and Sekiner, S. U. Design of wind farm layout using ant colony algorithm. Renewable Energy. 2012. 1: 53-62.

[4] Ronald, V. Book review: Computers and intractability: A guide to the theory of npcompleteness. Bull. Amer. Math. Soc. (N.S.). 1980. 1: 898-904.

[5] Lundberg, S. Performance Comparison of Wind Park Configurations. Technical Report Tech. Rep. 30R. Chalmers University of Technology, Department of Electric Power Engineering,. 2003.

[6] Ivanov, A.O. and Tuzhilin, A.A. Minimum networks: the Steiner problem and its generalizations. New York: CRC Press. 1994.

[7] Poteralski, A. Hybrid artificial immune strategy in identification and optimization of mechanical systems. J. Comput. Sci. 23 (2017), 216-225.

[8] Gilbert, E.N. and Pollak, H. O. Steiner minimum trees. SIAM 1. Appl. Math. 1968. 16: $1-29$.

[9] Corchado, L., Alonso, M. and Fyfe, C. Artificial immune systems: A novel paradigm to pattern recognition. In Artificial Neural Networks in Pattern Recognition. University of Paisley. 2002. 67-84.

[10] Melzak, Z.A. On the problem of steiner. Canad. Math. Bull. 1961. 4: 143-148.

[11] Tifroute, M. and Bouzahir, H. Optimization of cable layout design in a wind farm: a hybrid approach. Int. J. of Thermal Environmental Engineering. 2016. 11: 111-115.

[12] Prim, R.C. Shortest connection networks and some generalizations. Belltin System Technical Journal. 1957. 36: 1389-1401. 